\title{
Covariates streamline age-specific early life survival estimates of two chelonian species
}

\author{
D. Arsovski ${ }^{11}$, Olivier $^{2}$, X. Bonnet', S. Drilholle ${ }^{2}$, L. Tomovic ${ }^{3}$, A. Béchet ${ }^{2}$, A. Golubovic ${ }^{3}$ \& A. Besnard ${ }^{4}$ \\ 1 CEBC, UMR-7372, CNRS ULR, Villiers en Bois, France \\ 2 Institut de recherche de la Tour du Valat, Arles, France \\ 3 Institute of Zoology, Faculty of Biology, University of Belgrade, Belgrade, Serbia \\ 4 EPHE, CNRS, UM, SupAgro, IRD, INRA, UMR 5175 CEFE, PSL Research University, Montpellier, France
}

How to cite:

Arsovski D., Olivier A., Bonnet X., Drilholle S., Tomović L., Béchet A., Golubović A., Besnard A. 2018. Covariates streamline age-specific early life survival estimates of two chelonian species. Journal of Zoology [Internet] [cited 2018 Jul 11]. doi: 10.1111/jzo.12585

\section{Abstract}

Delayed maturity and high survival rates of immatures and adults characterize long-lived species' life histories. Understanding how these traits interact in stochastic environments is essential to understand early life in long-lived species and to assist conservation planning. Unfortunately, available informRödel of demographic traits in immatures are often fragmentary and the logistical difficulties of capturing and recapturing this cohort leave little room for improvement. Published immature chelonian survival estimates vary broadly, are often not age-specific, and lack precision. In an attempt to overcome this issue, we developed a novel modelling approach based on Capture-Recapture data to obtain robust age-specific survival probabilities in two chelonian species (the freshwater European pond turtle, Emys orbicularis, and the terrestrial Hermann's tortoise, Testudo hermanni). More specifically, we show that implementing a linear relationship between survival probabilities and age does not distort survival estimates and improves precision. Both species display a progressive increase in survival with age, reaching a plateau at the ages of four to five. As maturity occurs later in both species (8-12 years-old), the survival plateau might be governed by the final hardening of the carapace, which brings obvious survival benefits. Taking advantage of the flexibility of multievent models we encourage a systematic field approach even when very large samples from immatures cannot be amassed. Only so can demographic traits in chelonians, and more generally in long-lived species, be properly explored.

\section{Introduction}

Delayed sexual maturity, high imature and adult survival, and variation in the correlative nature of these traits define the life histories of long-lived animals (Dunham, Miles \& Reznick, 1988; Charnov, 1990; Congdon, Dunham \& van Loben, 1993; Congdon, Dunham \& van Loben Sels, 1994). Understanding their ecology, evolution and finally conservation biology requires robust demographic analyses based on thorough insight on these traits (Heppel, 1998; Enneson \& Litzgus, 2008). Nevertheless, developmental changes throughout a prolonged immature phase can induce huge variation in trait values; e.g. before (delayed) maturity mean annual survival increases linearly with age (see for instance Congdon et al., 1994; Jorgenson et al., 1997; Fernàndez-ChacOn et al., 2011; Hastings et al., 2011; DeLong et al., 2017). This complicates the understanding of trait covariation and mandates age-, or at least stage-specific estimates.

Longevity and delayed maturity are often expressed to the extreme in chelonians (Wilbur \& Morin, 1988). At present day, an ever-increasing set of threats (Klemens, 2000) have made that combination of life-history traits a chelonian Achilles' heel, with almost $70 \%$ of all 335 species threatened or recently extinct (almost $46 \%$ of which endangered or critically endangered); for many, conservation status has not been assessed (van Dijk et al., 2014). In order to counter this trend robust age-specific survival estimates are necessary to provide age-specific survival elasticities. Indeed, developing reliable projections of population growth using matrix models (Caswell, 2001), evaluating conservation priorities (Congdon et al., 1993; Heppel, 1998; Enneson \& Litzgus, 2008) and estimating conservation efficiency (e.g. after reintroduction and/or headstarting in Heppel, Crowder \& Crouse, 1996a; Mitrus, 2005; Canessa et al., 2016) rely on such endeavours in long-lived animals.

Alas, the elusive nature of chelonian early life precludes the amassing of large and dense CR (Capture-Recapture) datasets of aged immature turtles, actively hindering this goal. Although chelonian adult survival is relatively well-studied (Congdon et al., 1994; Henry et al., 1999; Chaloupka \& Limpus, 2005; Olivier et al., 2010; Bertolero et al., 2011; Bertolero, Pretus \& Oro, 2018), sporadic insight on early survival fails to paint a clear picture. Indeed, a rapid review of the sparse literature on agespecific early life chelonian survival revealed a wide spectrum of estimates ranging from $11 \%$ in neonate European pond turtles (Emys orbicularis) to $92 \%$ in juvenile Loggerhead turtles (Table 1). Much is likely a Alas, the elusive nature of chelonian early life precludes the amassing of large and dense CR (Capture-Recapture) datasets of aged immature turtles, actively hindering this goal. Although chelonian adult survival is relatively well-studied consequence of inherent interspecific differences; in juvenile tortoises survival estimates range from eight to $90 \%$, whereas in juvenile sea turtles they almost always exceed $70 \%$ (Table 1). Perhaps even more importantly, the plethora of methodological approaches (last column, Table 1) likely contributes largely to this variation (see also Pfaller et al., 2018). 
Tableau 1 Review of published chelonian age- or stage-specific survival estimates prior to maturity. Some studies reported ambiguous age categories here presented as immature. When explicitly reported, materials and methods are also provided

\begin{tabular}{|c|c|c|c|}
\hline Species & $\begin{array}{l}\text { Immature (age-specific) } \\
\text { survival }\end{array}$ & Materials \& methods & Reference \\
\hline \multicolumn{4}{|l|}{ Land tortoises } \\
\hline \multirow[t]{6}{*}{ T. h. hermanni } & Neonate: 0.39 f 0.10 & 19 years of CR, Cormack-Jolly-Seber & Fernandez-Chacbn et al. (2011) \\
\hline & 1-year-old: 0.45 f 0.08 & (CJS) model & \\
\hline & 2-year-old: 0.77 f 0.08 & & \\
\hline & 3-year-old: 0.68 f 0.07 & & \\
\hline & 4-year-old: 0.86 f 0.06 & & \\
\hline & 5-year-old: 0.88 f 0.05 & & \\
\hline \multirow[t]{3}{*}{ T. h. hermanni } & Neonate: 0.54 & 14 years of CR, CJS model & Bertolero (2002) \\
\hline & $1-2$ years-old: 0.64 & & \\
\hline & 3-9 years-old: 0.91 & & \\
\hline \multirow[t]{2}{*}{ T. h. hermanni } & $0-2$ years-old: 0.52 & 7 years of $\mathrm{CR}$ & Henry et \\
\hline & $3-9$ years-old: 0.88 & & \\
\hline Testudo graeca & Neonate: 0.39 & Thread trailing & Keller, Diaz-Paniagua \& Andreu (1998) \\
\hline Gopherus polyphemus & $1-4$ years-old: 0.45 & 1 & Wilson (1991) \\
\hline Gopherus polyphemus & $\begin{array}{l}\text { Immature: } 0.84 \\
\text { fFernandez-Chacon }\end{array}$ & $\begin{array}{l}12 \text { years of } \mathrm{CR} \text {, CJS open } \\
\text { population model }\end{array}$ & Tuberville et al. (2008) \\
\hline \multicolumn{4}{|l|}{ Freshwater turtles } \\
\hline \multirow{6}{*}{ Emys orbicularis } & Neonate: $0.02-0.08$ & 7 years of $\mathrm{CR}$, CJS model & Canessa et al. (2016) \\
\hline & 1 year-old: 0.53 & & \\
\hline & 2 years-old: 0.80 & & \\
\hline & $3-6$ years-old: $0.45-0.99$ & & \\
\hline & $7-11$ years-old: $0.55-1.15$ & & \\
\hline & $12+$ years old: $0.68-1.69$ & & \\
\hline \multirow[t]{2}{*}{ Emys orbicularis } & Neonate: 0.11 & 3 years of $\mathrm{CR}$ & Mitrus (2005) \\
\hline & 1-year-old: 0.64 & & \\
\hline Emys orbicularis & 1-year-old: 0.53 & / & Mitrus (2002) \\
\hline Emys orbicularis & $3-9$ years-old: 0.80 & l & Mitrus \& Zemanek (2004) \\
\hline Trachemys scripta & Immature: 0.83 & l & Frazer, Gibbons \& Greene (1990) \\
\hline Myuchelys georgesi & Immature: 0.58 & / & Blamires et al. (2005) \\
\hline Chelydra serpentina & Immature: 0.75 & l & Cunnington \& Brooks (1996) \\
\hline Chelydra serpentina & Immature: $0.65-0.82$ & / & Congdon et al. (1994) \\
\hline Chrysemys picta & Immature: 0.81 & l & Wilbur (1975) \\
\hline Chrysemys picta & Immature: 0.83 & l & Mitchell (1988) \\
\hline Emydoidea blandingii & Immature: 0.78 & l & Congdon et al. (1993) \\
\hline Kinosternon flavescens & Immature: 0.64 & l & Iverson (1991) \\
\hline Kinosternon subrubrum & Immature: 0.72 & 1 & Frazer, Gibbons \& Greene (1991) \\
\hline Clemmys guttata & Neonate: 0.81 & 30 years of CR, CJS model & Enneson \& Litzgus, 2008; \\
\hline \multicolumn{4}{|l|}{ Marine turtles } \\
\hline Caretta caretta & Immature: 0.72 & Catch-curve analysis & Bjorndal, Bolten \& Martins (2003b) \\
\hline Caretta caretta & Immature: 0.70 & Catch-curve analysis & Frazer (1987) \\
\hline Caretta caretta & Immature: 0.89 & Catch-curve analysis & Epperly et al. (2001) \\
\hline Caretta caretta & Immature: 0.83 & CJS model & Heppell et al. (1996b) \\
\hline Caretta caretta & Immature: 0.86/0.92 & CJS model & Chaloupka \& Limpus (2002) \\
\hline \multirow{4}{*}{ Chelonia mydas } & 1-year-old: 0.91 f 0.03 & 23 years of $C R$, Burnham model & Bjorndal, Bolten \& Chaloupka (2003a) \\
\hline & 2-year-old: 0.85 f 0.05 & & \\
\hline & 3-year-old: 0.91 f 0.04 & & \\
\hline & 4-year-old: 0.76 f 0.03 & & \\
\hline Chelonia mydas & Immature: $0.88 / 0.85$ & CJS model & Chaloupka \& Limpus (2005) \\
\hline Chelonia mydas & Immature: 0.83 & 13 years of CR, CJS model & Patricio et al. (2011) \\
\hline Chelonia mydas & Immature: 0.88 f 0.02 & 9 years of $\mathrm{CR}, \mathrm{CJS}$ model & Chaloupka \& Limpus (2005) \\
\hline
\end{tabular}


Here, we present a general analytical framework that allows for age-specific survival estimates to be obtained from sparse CR data (i.e. small and varying numbers of individuals per age class). We applied this approach on two distantly related (within Testudinoidea in Crawford et al., 2014), Near Threatened (IUCN 2017) chelonians: the European pond turtle and the Hermann's tortoise (Testudo hermanni). In T. h. hermanni (the western subspecies) age-specific survival progressively increases with age from 39\% in neonates to $97 \%$ in 6-year olds and older (Fernandez-Chacon et al., 2011); juvenile survival estimates of its eastern counterpart (T. h. boettgeri) are lacking. Immature European pond turtle survival exhibits a very wide range (11\% to $90 \%$ in Table 1$)$, and robust age-specific analyses are lacking.

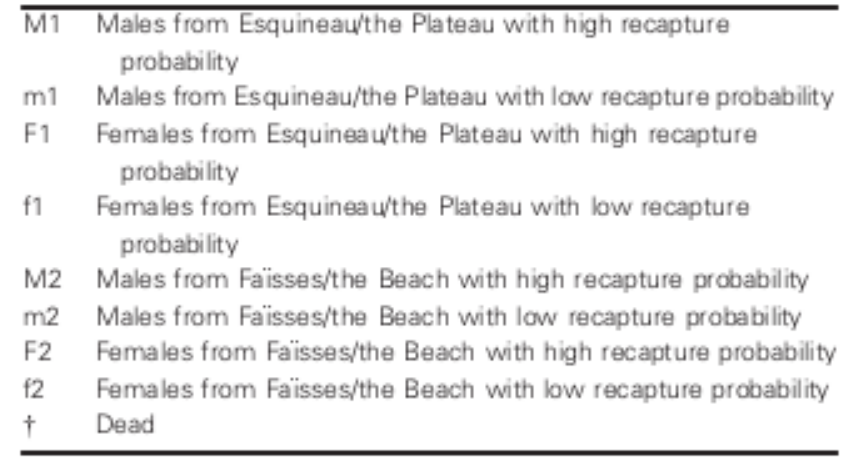

Tableau 2 Abbreviations (left column) and descriptions (right column) of states that European pond turtles from Camargue (Esquineau and Faïsses), France and Hermann's tortoises from Golem Grad (the Plateau and the Beach), Macedonia can assume and transit among

\section{Materials and methods}

\section{Studied species and sites European pond turtle}

The European pond turtle inhabits a broad range of habitats across Europe, northern Africa and westerns Asia. Nevertheless, it is often locally endangered and even extinct (Fritz \& Chiari, 2013). The European pond turtle is largely impacted by diverse anthropogenic factors such as habitat loss, regulation of rivers and shoreline development that homogenize habitats and fragment populations (Rogner, 2009).

During 16 years (1997-2012), a total sampling hand-capture effort of $598 \mathrm{~h}$ and 6158 trap-days in the Natural Reserve of Tour du Valat (Camargue, France, 250 ha $43^{\circ} 30^{\circ} \mathrm{N}, 4^{\circ} 40^{\circ} \mathrm{E}$ ) resulted in 749 captured European pond turtles (for details on field methodology see Olivier et al., 2010 and Ficheux et al., 2014). Among these, 298 could be aged using growth-rings (Castanet, 1988; Olivier, 2002) for a total of 809 captures. Sex was assigned for sexually dimorphic individuals followed by permanent notch marking on the carapace (Cagle, 1939).

\section{Hermann's tortoise}

Hermann's tortoises are widespread across the European Mediterranean region. Nevertheless, the westerns subspecies has suffered major losses in habitat and therefore distribution range (Stubbs, 1995; Bertolero et al., 2011). With rapid uncontrolled urbanization in the Balkans, its eastern counterpart might be heading for a similar fate. Local illegal and legal tortoise harvest of huge proportions in the past decades (Cites trade report on Testudo hermanni, 2017) have massively contributed to this trend.

During 9 years (2008-2016), we studied a very dense ( $\sim 60$ adults per hectare, Bonnet et al., 2016) population on Golem Grad Island (Prespa Lake, Macedonia, 18 ha, 40 $52^{\circ} 09^{\prime \prime} \mathrm{N}, 20^{\circ} 59^{\circ} 17^{\prime \prime} \mathrm{E}$ ). Each year the area was intensively patrolled (2-12 observers). Overall, 169 search days enabled us to capture 1830 individuals. Among them, 466 could be aged at 1225 capture occasions. Every captured individual was sexed when sexually dimorphic, aged, and permanently marked using a notche-code on the marginal-scutes (Cagle, 1939). In the course of recaptures, age was re-estimated blindly (for error rate and more details on ageing and general CR methodology see Bonnet et al., 2016 and Arsovski et al., 2018).

\section{Multievent model design}

We performed goodness-of-fit tests on the datasets based on the CJS model. Attributed to the detection of trap dependence and transience (see Results) that might be induced either by age-specific variations of recapture and survival probability but also by capture heterogeneity (Olivier, 2002; Ficheux et al., 2014), we used multievent modelling (Pradel, 2005). Events are field observations masking possibly multiple states; multievent models estimate the probability of observing a state given the event it is masked under using hidden Markov chains. Accordingly, sex was coded as a state in order to deal with sex uncertainty in young individuals. In order to deal with potential heterogeneity in recapture rates, we built mixture models (Pledger, Pollock \& Norris, 2003) in which individuals can belong to two classes of individuals exhibiting different recapture probabilities (see for instance Prévot-Julliard, Lebreton \& Pradel, 1998 for such a procedure).

Our models were based on nine underlying biological states (Table 2), i.e. a combination of three variables: sex, site (Esquineau and Baisses for European pond turtles; Plateau and Beach for Hermann's tortoises) and recapture heterogeneity class, and a dead state. At first capture, individuals depart from all states but dead. Subsequently, they can annually transit between 
states following five successive transition matrices: survival from $t$ to $t+1$ (matrix $\Phi$, Table 3a), dispersal between sites, given the individual survived (matrix $\Psi$, Table $3 \mathrm{~b}$ ), and change of recapture heterogeneity class given

Tableau 3 Elementary matrices describing transitions among elementary states (' $\mathrm{m} / \mathrm{M}$ ' and ' $\mathrm{f} / \mathrm{F}$ ' refer to males and females, respectively, capitals denoting high recapture probability and vice versa; ' 1 ' and ' 2 ' refer to the two sites of both datasets: Esquineau/the Plateau and Faïsses/the Beach, respectively; Table 2): (a) survival matrix 0, / is the probability to survive from t to $t+1$; (b) dispersal matrix $\Psi$, $w$ is the probability to stay faithful to the site of first capture from $t$ to $t+1$, whereas after a dispersal event the elementary state is prefixed a ' $d$ ' (e.g. M1 -> dM1; (c) change of heterogeneity class matrix F, $f$ is the probability of reaching the high recapture probability class; (d) first event matrix $\mathbf{P}, \mathbf{p}$ is the probability of being recaptured after which the departure state is prefixed a ' $c$ ', whereas when failed to be recaptured the prefix is ' 0 '; (e) second event matrix $S$, $\mathrm{s}$ is the probability to assess the sex of the individual, 0 - unobserved, 1 - observed as male in Esquineau/on the Plateau, 2 observed as female in Esquineau/on the Plateau, 3 - observed as unsexable in Esquineau/on the Plateau, 4 - observed as male in Faïsses/on the Beach, 5 - observed as female in Faïsses/on the Beach and 6 - observed as unsexable in Faïsses/on the Beach

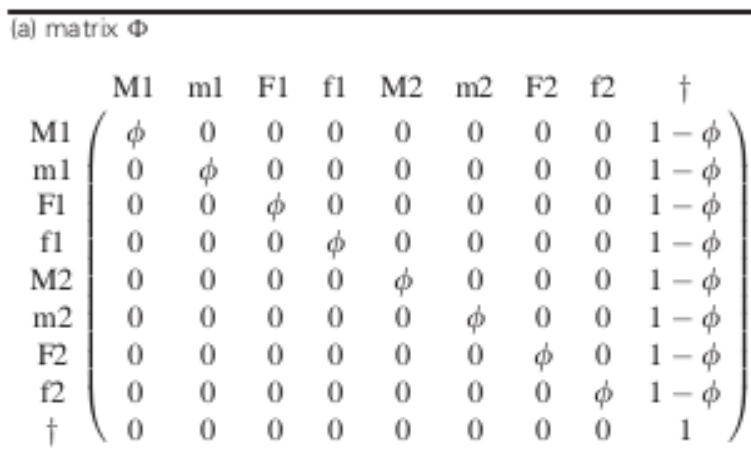

(b) matrix $\Psi$

\begin{tabular}{|c|c|c|c|c|c|c|c|c|c|c|c|c|c|c|c|c|}
\hline M1 & $\mathrm{ml}$ & F1 & f1 & M2 & $\mathrm{m} 2$ & $\mathrm{~F} 2$ & f2 & $\mathrm{dM} 1$ & $\mathrm{dm} 1$ & $\mathrm{dF} 1$ & df 1 & $\mathrm{dM} 2$ & $\mathrm{dm} 2$ & $\mathrm{dF} 2$ & df 2 & $\dagger$ \\
\hline M1 $(\psi$ & 0 & 0 & 0 & 0 & 0 & 0 & 0 & $1-\psi$ & 0 & 0 & 0 & 0 & 0 & 0 & 0 & $1-\phi$ \\
\hline $\mathrm{m} 1$ & $\psi$ & 0 & 0 & 0 & 0 & 0 & 0 & 0 & $1-\psi$ & 0 & 0 & 0 & 0 & 0 & 0 & $1-\phi$ \\
\hline $\mathrm{F} 1$ & 0 & $\psi$ & 0 & 0 & 0 & 0 & 0 & 0 & 0 & $1-\psi$ & 0 & 0 & 0 & 0 & 0 & $1-\phi$ \\
\hline fl & 0 & 0 & $\psi$ & 0 & 0 & 0 & 0 & 0 & 0 & 0 & $1-\psi$ & 0 & 0 & 0 & 0 & $1-\phi$ \\
\hline M2 & 0 & 0 & 0 & $\psi$ & 0 & 0 & 0 & 0 & 0 & 0 & 0 & $1-\psi$ & 0 & 0 & 0 & $1-\phi$ \\
\hline $\mathrm{m} 2$ & 0 & 0 & 0 & 0 & $\psi$ & 0 & 0 & 0 & 0 & 0 & 0 & 0 & $1-\psi$ & 0 & 0 & $1-\phi$ \\
\hline $\mathrm{F} 2$ & 0 & 0 & 0 & 0 & 0 & $\psi$ & 0 & 0 & 0 & 0 & 0 & 0 & 0 & $1-\psi$ & 0 & $1-\phi$ \\
\hline $\mathrm{f} 2$ & 0 & 0 & 0 & 0 & 0 & 0 & $\psi$ & 0 & 0 & 0 & 0 & 0 & 0 & 0 & $1-\psi$ & $1-\phi$ \\
\hline 0 & 0 & 0 & 0 & 0 & 0 & 0 & 0 & 0 & 0 & 0 & 0 & 0 & 0 & 0 & 0 & 1 \\
\hline
\end{tabular}

(c) matrix $F$

\begin{tabular}{|c|c|c|c|c|c|c|c|c|}
\hline & M1 & $\mathrm{m} 1$ & F1 & f1 & M2 & $\mathrm{m} 2$ & $\mathrm{~F} 2$ & f2 \\
\hline M1 & 1 & 0 & 0 & 0 & 0 & 0 & 0 & 0 \\
\hline $\mathrm{m} 1$ & 0 & 1 & 0 & 0 & 0 & 0 & 0 & 0 \\
\hline $\mathrm{F} 1$ & 0 & 0 & 1 & 0 & 0 & 0 & 0 & 0 \\
\hline f1 & 0 & 0 & 0 & 1 & 0 & 0 & 0 & 0 \\
\hline M2 & 0 & 0 & 0 & 0 & 1 & 0 & 0 & 0 \\
\hline $\mathrm{m} 2$ & 0 & 0 & 0 & 0 & 0 & 1 & 0 & 0 \\
\hline $\mathrm{F} 2$ & 0 & 0 & 0 & 0 & 0 & 0 & 1 & 0 \\
\hline $\mathrm{f} 2$ & 0 & 0 & 0 & 0 & 0 & 0 & 0 & 1 \\
\hline dM1 & 0 & 0 & 0 & 0 & $f$ & $1-f$ & 0 & 0 \\
\hline $\mathrm{dm} 1$ & 0 & 0 & 0 & 0 & $f$ & $1-f$ & 0 & 0 \\
\hline $\mathrm{dF} 1$ & 0 & 0 & 0 & 0 & 0 & 0 & $f$ & $1-f$ \\
\hline df 1 & 0 & 0 & 0 & 0 & 0 & 0 & $f$ & $1-f$ \\
\hline $\mathrm{dM} 2$ & $f$ & $1-f$ & 0 & 0 & 0 & 0 & 0 & 0 \\
\hline $\operatorname{dm} 2$ & $f$ & $1-f$ & 0 & 0 & 0 & 0 & 0 & 0 \\
\hline $\mathrm{dF} 2$ & 0 & 0 & $f$ & $1-f$ & 0 & 0 & 0 & 0 \\
\hline df 2 & 0 & 0 & $f$ & $1-f$ & 0 & 0 & 0 & 0 \\
\hline$\dagger$ & 0 & 0 & 0 & 0 & 0 & 0 & 0 & 0 \\
\hline
\end{tabular}




\begin{tabular}{|c|c|c|c|c|c|c|c|c|c|c|c|c|c|c|c|c|c|}
\hline & $0 \mathrm{M} 1$ & $0 \mathrm{ml}$ & $0 \mathrm{~F} 1$ & $0 \mathrm{f} 1$ & $0 \mathrm{M} 2$ & $0 \mathrm{~m} 2$ & $0 \mathrm{~F} 2$ & Of 2 & cM1 & $\mathrm{cm} 1$ & $\mathrm{cF} 1$ & cf 1 & $\mathrm{cM} 2$ & $\mathrm{~cm} 2$ & $\mathrm{cF} 2$ & cf 2 & $\dagger$ \\
\hline M1 & $(1-p$ & 0 & 0 & 0 & 0 & 0 & 0 & 0 & $p$ & 0 & 0 & 0 & 0 & 0 & 0 & 0 & $0)$ \\
\hline $\mathrm{m} 1$ & 0 & $1-p$ & 0 & 0 & 0 & 0 & 0 & 0 & 0 & $p$ & 0 & 0 & 0 & 0 & 0 & 0 & 0 \\
\hline $\mathrm{F} 1$ & 0 & 0 & $1-p$ & 0 & 0 & 0 & 0 & 0 & 0 & 0 & $p$ & 0 & 0 & 0 & 0 & 0 & 0 \\
\hline $\mathrm{f} 1$ & 0 & 0 & 0 & $1-p$ & 0 & 0 & 0 & 0 & 0 & 0 & 0 & $p$ & 0 & 0 & 0 & 0 & 0 \\
\hline M2 & 0 & 0 & 0 & 0 & $1-p$ & 0 & 0 & 0 & 0 & 0 & 0 & 0 & $p$ & 0 & 0 & 0 & 0 \\
\hline $\mathrm{m} 2$ & 0 & 0 & 0 & 0 & 0 & $1-p$ & 0 & 0 & 0 & 0 & 0 & 0 & 0 & $p$ & 0 & 0 & 0 \\
\hline $\mathrm{F} 2$ & 0 & 0 & 0 & 0 & 0 & 0 & $1-p$ & 0 & 0 & 0 & 0 & 0 & 0 & 0 & $p$ & 0 & 0 \\
\hline $\mathrm{f} 2$ & 0 & 0 & 0 & 0 & 0 & 0 & 0 & $1-p$ & 0 & 0 & 0 & 0 & 0 & 0 & 0 & $p$ & 0 \\
\hline$\dagger$ & 0 & 0 & 0 & 0 & 0 & 0 & 0 & 0 & 0 & 0 & 0 & 0 & 0 & 0 & 0 & 0 & 1) \\
\hline
\end{tabular}

(e) matrix $S$

$\left.\begin{array}{c|ccccccc}0 \mathrm{M} 1 & 0 & 1 & 2 & 3 & 4 & 5 & 6 \\ 0 \mathrm{~m} 1 & 0 & 0 & 0 & 0 & 0 & 0 \\ 0 \mathrm{~F} 1 & 1 & 0 & 0 & 0 & 0 & 0 & 0 \\ \text { Of } 1 & 1 & 0 & 0 & 0 & 0 & 0 & 0 \\ 0 \mathrm{M} 2 & 1 & 0 & 0 & 0 & 0 & 0 & 0 \\ 0 \mathrm{~m} 2 & 1 & 0 & 0 & 0 & 0 & 0 & 0 \\ \text { OF2 } & 1 & 0 & 0 & 0 & 0 & 0 & 0 \\ \text { Of } 2 & 1 & 0 & 0 & 0 & 0 & 0 & 0 \\ \mathrm{cM} 1 & 1 & 0 & 0 & 0 & 0 & 0 & 0 \\ \mathrm{~cm} 1 & 0 & s & 0 & 1-s & 0 & 0 & 0 \\ \mathrm{cF} 1 & 0 & s & 0 & 1-s & 0 & 0 & 0 \\ \mathrm{cf} 1 & 0 & 0 & s & 1-s & 0 & 0 & 0 \\ \mathrm{cM} 2 & 0 & 0 & s & 1-s & 0 & 0 & 0 \\ \mathrm{~cm} 2 & 0 & 0 & 0 & 0 & s & 0 & 1-s \\ \mathrm{cF} 2 & 0 & 0 & 0 & 0 & s & 0 & 1-s \\ \mathrm{c} 2 & 0 & 0 & 0 & 0 & 0 & s & 1-s \\ \dagger & 0 & 0 & 0 & 0 & 0 & s & 1-s \\ 1 & 0 & 0 & 0 & 0 & 0 & 0\end{array}\right)$

the individual survived and changed site (matrix F, Table 3c). The approach was simpler on the Hermann's tortoises, not parameterizing matrices $\psi$ and $F$ since individuals did not change sites, nor were caught in traps. Note that for easier implementation, $\psi$ is a 9*17 matrix where eight departure states can transit to a "stay" or "moved" state. F is thus a $17 * 9$ matrix, that allows "moved" individuals to change recapture probability class (since we suspect recapture heterogeneity to be related to trap location we assumed that "stay" individuals would not change recapture heterogeneity class). Finally, individuals can be recaptured or not. For easier implementation, this event matrix was split into two matrices, one allowing to model recapture probability given the states (matrix $\mathrm{P}$, Table 3d), the other to estimate the probability of correctly sexing individuals (matrix S, Table 3e).

\section{Building biological scenarios}

Since young and small European pond turtles are less mobile and therefore less likely to be captured in a trap, two age classes were considered when modelling recapture probability (additional explorations would require a bigger sample): neonateto-2-year-olds and older than 2 years. Initially, survival-atage was a linear covariate with a plateau at age six. The more numerous tortoise dataset allowed multiple combinations of age-recapture probability to be explored, while maintaining an age effect on survival. Subsequently, in both species we explored multiple additive relationships with age, site, sex, time and capture heterogeneity.

Afterwards, maintaining the best parametrization of recapture probabilities we explored the effects of sex and site on survival. Lastly, we explored different parametrizations of the age effect on survival probabilities, namely linear, logarithmic and quadratic versus discrete, and different age at plateau. We superimpose our results from the best-fit discrete model against those aided by covariates and note the possible benefits and drawbacks. All models were implemented in E-SURGE (Choquet, Rouan \& Pradel, 2009) separately per species, ranking competitive models using Akaike information criteria (AIC, Burnham \& Anderson, 2002). 
Table 4 Breakdown of numbers of captured European pond turtles from Camargue, France and Hermann's tortoises from Golem Grad island, Macedonia per hatching-year

$\begin{array}{lcc}\text { Hatching-year } & \text { European pond turtle } & \text { Hermann's tortoise } \\ 1990 & 3 & / \\ 1991 & 6 & / \\ 1992 & 11 & / \\ 1993 & 12 & / \\ 1994 & 18 & / \\ 1995 & 11 & / \\ 1996 & 4 & / \\ 1997 & 6 & / \\ 1998 & 16 & 7 \\ 1999 & 16 & 10 \\ 2000 & 16 & 16 \\ 2001 & 6 & 14 \\ 2002 & 4 & 34 \\ 2003 & 16 & 36 \\ 2004 & 26 & 41 \\ 2005 & 26 & 44 \\ 2006 & 28 & 64 \\ 2007 & 36 & 87 \\ 2008 & 15 & 48 \\ 2009 & 15 & 12 \\ 2010 & 4 & 5 \\ 2011 & 3 & 19 \\ 2012 & / & 15 \\ 2013 & / & 7 \\ 2014 & / & 7 \\ 2015 & / & \end{array}$

\section{Results}

All 298 European pond turtles had hatched between 1990 and 2011, and all 466 Hermann's tortoises between 1999 and 2015. The chronological breakdown of captured animal numbers per hatching year can be found in Table 4. In both data-sets the goodnessof-fit tests were significant $\left(\mathrm{chi}^{2}=127.35\right.$ and 72.09 , d.f. $=60$ and 31 , respectively, $\left.\mathrm{P}<0.001\right)$ with significant transience and trap-dependence (both $\mathrm{P}<0.001$ ), suggesting strong survival or recapture heterogeneity.

\section{European pond turtle}

The best-fitted model (Table 5) implied an additive time variation of both age classes' recapture probabilities, the second (>2 years old) also exhibiting heterogeneity and sex differences. Removing the effect of recapture heterogeneity increases AIC sharply (by 82.6), confirming its importance. The younger age-class displayed similar capture probabilities as the low heterogeneity class of the older age class (on average ranging between 0.23 and 0.34 , between capture occasions). Higher heterogeneity class males were consistently higher than the respective females, with average respective estimates of 0.84 and 0.72 . Survival retained the linear age-effect and did not vary between sites and sexes. Exploring a survival age-plateau resulted with two very

close models $($ DAIC $=0.36$ ), with survival summiting at 4 and 5 years of age. Nevertheless, neonate survival estimates are significantly different $(0.10 \mathrm{f} 0.05$ vs. $0.20 \mathrm{f} 0.07)$; the discrete model was unable to provide these estimates due to a lack of neonates. All three models demonstrate a steep increase in survival probability between zero and 4 years of age (Fig. 1). The differences in survival probabilities between the discrete and covariate models we re low, ranging between one and nine percent among age-classes. Nevertheless, the discrete model was much less precise (Fig. 1).

\section{Hermann's tortoise}

After model selection, regarding recapture probability 1- to 5-year-olds formed a separate cohort whereas all further age classes were considered separately. All are site dependent, while only those from the Plateau experience recapture heterogeneity (lowering AIC by 71). Among them, the higher heterogeneity class ranged between 0.56 and 0.71 , the low 
heterogeneity class from 0.01 to 0.07 and Beach tortoises somewhere in the middle between 0.32 and 0.40 . All exhibit additive time variation. Exploring different parameterizations of the age-effect on survival resulted with a logarithmic to linear relationship reaching a plateau at 4-6 years of age, or a discrete model summiting at the age of four [DAIC between these models ranged between 0.6 and 1.3, Table 5; models whose DAIC from the best fit model $<2$ are considered to also have substantial support (Burnham \& Anderson, 2002)]. Despite lowering AIC, adding site and/or sex effects on discrete model survival failed to provide precise estimates, thus the age-effect discrete model was used for comparison purposes. With covariates, best model fit was achieved when female survival from the Plateau was considered separately. Plateau female survival probabilities increased linearly with age from 0.08 when neonate to 0.86 when 5 -years-old. Conversely, male tortoises and females from the Beach expressed significantly higher survival at all ages starting at 0.32 with a plateau at 0.97 (Fig. 2). Superimposing the discrete model estimates (despite being slightly lower due to lack of separation of Plateau females) showed a broad overlap of confidence intervals but was unable to estimate neonate $(\mathrm{N}=1)$ survival (Fig. 2).

\section{Discussion}

By means of advanced multievent CR modelling, this study demonstrates that even sparse datasets can be used to obtain agespecific estimates of survival in long-lived species. Consequently, it demonstrates that, as in other long-lived vertebrates, the substantially prolonged immature phase of both the Hermann's tortoise and the European pond turtle entail a steep and progressive increase in survival with age.

The age-linear covariate (continuous) models are streamlined versions of their discrete counterparts, suggesting no bias in the age-specific survival estimates. Moreover, the continuous models clearly outperformed the discrete models in terms of precision, especially in the sparser dataset of the European

\begin{tabular}{|c|c|c|c|c|c|c|}
\hline \multirow[b]{2}{*}{$r$} & \multicolumn{2}{|l|}{ Model definition } & \multirow[b]{2}{*}{$k$} & \multirow[b]{2}{*}{ Dev. } & \multirow[b]{2}{*}{$\mathrm{AlC}$} & \multirow[b]{2}{*}{$w$} \\
\hline & Survival & Recapture & & & & \\
\hline \multicolumn{7}{|c|}{ European pond turtle } \\
\hline 12 & Lin_6*sex*site & lage $\leq 2^{*}$ site; age $>2^{*} \mathrm{H}^{*}$ site*sex] + year & 44 & 2608.0 & 2696.0 & 0.0 \\
\hline 17 & Lin_6*sex*site & lage $\leq 2 * \mathrm{H}$; age $>2 * \mathrm{H}^{*}$ site* sex] + year & 44 & 2610.1 & 2698.1 & 0.0 \\
\hline 13 & Lin_6* ${ }^{*} \mathrm{sex}^{*}$ site & lage $\leq 2$; age $>2 * H^{*}$ site* sex] + year & 43 & 2610.6 & 2696.6 & 0.0 \\
\hline 21 & Lin_6*sex*site & age $\leq 2 ;$ age $>2 * H^{*}$ site* sex & 29 & 2647.0 & 2705.0 & 0.0 \\
\hline 23 & Lin_6* sex*site & [age $\leq 2$; age $>2 *$ site*sex] + year & 35 & 2708.5 & 2778.5 & 0.0 \\
\hline 14 & Lin_6*sex*site & [age $\leq 2 ;$ age $>2 * \mathrm{H}^{*}$ site] + year & 39 & 2619.0 & 2697.0 & 0.0 \\
\hline 11 & Lin_6*sex*site & lage $\leq 2$; age $>2 * \mathrm{H}^{*}$ sex] + year & 39 & 2617.9 & 2696.0 & 0.0 \\
\hline 15 & Lin_6*sex*site & lage $\leq 2 ;$ age $>2 * \mathrm{H}]+$ year & 37 & 2623.5 & 2697.5 & 0.0 \\
\hline 8 & Lin_6*sex & lage $\leq 2 ;$ age $>2 * \mathrm{H}^{*}$ sex] + year & 37 & 2618.5 & 2692.5 & 0.0 \\
\hline 6 & Lin_6*site & lage $\leq 2$; age $>2 * \mathrm{H}^{*}$ sex] + year & 37 & 2618.4 & 2692.4 & 0.0 \\
\hline 5 & Lin_6 & lage $\leq 2 ;$ age $>2 * \mathrm{H}^{*}$ sex] + year & 36 & 2618.7 & 2690.7 & 0.1 \\
\hline 4 & $\log _{-} 6$ & lage $\leq 2$; age $>2 * \mathrm{H}^{*}$ sex] + year & 36 & 2618.6 & 2690.6 & 0.1 \\
\hline 22 & Ouad_6 & lage $\leq 2$; age $>2 * \mathrm{H}^{*}$ sex] + year & 36 & 2633.7 & 2705.7 & 0.0 \\
\hline 1 & Lin_5 & lage $\leq 2$; age $>2 * \mathrm{H}^{*}$ sex] + year & 36 & 2614.7 & 2686.7 & 0.4 \\
\hline 3 & Log_5 & lage $\leq 2 ;$ age $>2 * \mathrm{H}^{*}$ sex] + year & 36 & 2618.0 & 2690.0 & 0.1 \\
\hline 19 & Ouad_5 & [age $\leq 2$; age $>2 * \mathrm{H}^{*}$ sex] + year & 36 & 2629.7 & 2701.7 & 0.0 \\
\hline 2 & Lin_4 & lage $\leq 2$; age $>2 * \mathrm{H}^{*}$ sex] + year & 36 & 2615.1 & 2687.1 & 0.3 \\
\hline 9 & $\log _{-} 4$ & lage $\leq 2$; age $>2 * \mathrm{H}^{*}$ sex] + year & 36 & 2621.2 & 2693.2 & 0.0 \\
\hline 18 & Quad_4 & lage $\leq 2$; age $>2 * \mathrm{H}^{*}$ sex] + year & 36 & 2626.1 & 2698.1 & 0.0 \\
\hline 16 & Lin_3 & lage $\leq 2 ;$ age $>2 * \mathrm{H}^{*}$ sexl + year & 36 & 2625.6 & 2697.6 & 0.0 \\
\hline 20 & Log_3 & lage $\leq 2 ;$ age $>2 * \mathrm{H}^{*}$ sex] + year & 36 & 2631.7 & 2703.7 & 0.0 \\
\hline 10 & Quad_3 & lage $\leq 2$; age $>2 * \mathrm{H}^{*}$ sex] + year & 36 & 2623.7 & 2695.7 & 0.0 \\
\hline 7 & Age & lage $\leq 2$; age $>2 * \mathrm{H}^{*}$ sex] + year & 41 & 2609.3 & 2691.3 & 0.0 \\
\hline \multicolumn{7}{|c|}{ Hermann's tortoise } \\
\hline 53 & Age & age & 33 & 4360.2 & 4426.2 & 0.0 \\
\hline 43 & Age & age*year & 155 & 3966.0 & 4276.0 & 0.0 \\
\hline 37 & Age & age + year & 48 & 4015.5 & 4111.5 & 0.0 \\
\hline 57 & Age & [age0; 1-9] + year & 32 & 4510.6 & 4574.6 & 0.0 \\
\hline 55 & Age & lage0; 1,2-9] + year & 34 & 4473.1 & 4541.1 & 0.0 \\
\hline 54 & Age & [age0; 1-2;3-9] + year & 34 & 4412.3 & 4480.3 & 0.0 \\
\hline 51 & Age & lage0; 1-3;4-9] + year & 34 & 4345.2 & 4413.2 & 0.0 \\
\hline 50 & Age & [age0; $1-4 ; 5-9]+$ year & 34 & 4290.1 & 4358.1 & 0.0 \\
\hline 48 & Age & lage0; 1-5;6-9] + year & 34 & 4227.1 & 4295.1 & 0.0 \\
\hline 41 & Age & [age0; 1-6;7-9] + year & 34 & 4160.3 & 4228.3 & 0.0 \\
\hline 40 & Age & [age0; 1-7;8-9] + year & 34 & 4156.8 & 4224.8 & 0.0 \\
\hline 42 & Age & [age0; 1-8;9] + year & 34 & 4193.6 & 4261.6 & 0.0 \\
\hline 36 & Age & lage0; $1-3 ; 4 ; 5 ; 6 ; 7 ; 8 ; 9]+$ year & 46 & 4019.2 & 4111.2 & 0.0 \\
\hline 46 & Age & [age0; 1-2;3-5;6-9] + year & 36 & 4223.0 & 4295.0 & 0.0 \\
\hline 52 & Age & lage0; $1 ; 2 ; 3 ; 4-9]$ + year & 38 & 4341.2 & 4417.2 & 0.0 \\
\hline 45 & Age & lage0; $1 ; 2-3 ; 4-5 ; 6-9]+$ year & 38 & 4213.0 & 4289.0 & 0.0 \\
\hline 47 & Age & lage0; 1-2;3-5;6-9] + year & 36 & 4223.0 & 4295.0 & 0.0 \\
\hline 49 & Age & lage0; 1-5;6-9] + year & 34 & 4227.1 & 4295.1 & 0.0 \\
\hline 56 & Age & [age0; 1,2;3-9] + year & 34 & 4473.1 & 4541.1 & 0.0 \\
\hline 38 & Age & lage0; $1-5 ; 6 ; 7 ; 8 ; 9]+$ year & 40 & 4033.5 & 4113.5 & 0.0 \\
\hline 44 & Age & lage0; 1-3;4-5;6-9] + year & 36 & 4216.7 & 4288.7 & 0.0 \\
\hline 39 & Age & lage0; $(1-5 ; 6 ; 7 ; 8 ; 9) *$ sex] + year & 45 & 4029.7 & 4119.7 & 0.0 \\
\hline 29 & Age & lage0; $(1-5 ; 6 ; 7 ; 8 ; 9) *$ site] + year & 45 & 3947.7 & 4037.7 & 0.0 \\
\hline 31 & Age & lage0; $\left.[1-5 ; 6 ; 7 ; 8 ; 9)^{*} \mathrm{H}\right]+$ year & 50 & 3942.5 & 4042.5 & 0.0 \\
\hline 30 & Age & lage0; $(1-5 ; 6 ; 7 ; 8 ; 9) *$ site*H] + year & 60 & 3920.1 & 4040.1 & 0.0 \\
\hline 25 & Age & lage0; $\{1-5 ; 6 ; 7 ; 8 ; 9)^{*}$ site $\left.\left(P^{*} H\right)\right]+$ year & 53 & 3926.6 & 4032.6 & 0.0 \\
\hline 33 & Age & lage0; $(1-5 ; 6 ; 7 ; 8 ; 9) *$ sex*site*H] + year & 80 & 3899.4 & 4059.4 & 0.0 \\
\hline 35 & Age & [age0; 1-5;(6;7;8;9)*site $(\mathrm{P} * \mathrm{H})]+$ year & 51 & 3997.0 & 4099.0 & 0.0 \\
\hline
\end{tabular}

Table 5 Model selection procedure showing all competing models for both species in order of conception: $r=$ model rank; $k=$ number of parameters; Dev. = residual deviance; $\mathrm{AICc}=$ Akaike information criteria; $\mathbf{w}=\mathbf{A I C c}$ relative weight; age = discrete model; $\mathbf{H}$ = capture heterogeneity; $\mathbf{P}$ = Plateau; Lin, Quad; Log = linear, quadratic, logarithmic covariate, respectively, on the age- 


\begin{tabular}{|c|c|c|c|c|c|c|}
\hline \multirow[b]{2}{*}{$r$} & \multicolumn{2}{|l|}{ Model definition } & \multirow[b]{2}{*}{$\mathrm{k}$} & \multirow[b]{2}{*}{ Dev. } & \multirow[b]{2}{*}{ AIC } & \multirow[b]{2}{*}{ w } \\
\hline & Survival & Recapture & & & & \\
\hline$\overline{28}$ & Lin & [age0; $(1-5 ; 6 ; 7 ; 8 ; 9)^{*}$ site $\left.\left(P^{*} H\right)\right]+$ year & 47 & 3943.6 & 4037.6 & $\overline{0.0}$ \\
\hline 34 & Quad & [age $0 ;(1-5 ; 6 ; 7 ; 8 ; 9)^{*}$ site $\left.\left(P^{*} H\right)\right]+$ year & 47 & 3973.9 & 4067.9 & 0.0 \\
\hline 21 & $\log$ & [age $0 ;(1-5 ; 6 ; 7 ; 8 ; 9)^{*}$ site $\left.\left(P^{*} H\right)\right]+$ year & 47 & 3936.9 & 4030.9 & 0.0 \\
\hline 24 & Age_8 & [age0; $(1-5 ; 6 ; 7 ; 8 ; 9)^{*}$ site $\left.\left(P^{*} H\right)\right]+$ year & 52 & 3928.3 & 32.3 & 0.0 \\
\hline 2 & Lin_8 & {$\left[\right.$ age $0 ;(1-5 ; 6 ; 7 ; 8 ; 9)^{*}$ site $\left.\left(P^{*} H\right)\right]+$ year } & 46 & 3940.8 & 4032.8 & 0.0 \\
\hline 16 & Log_8 & [age $0 ;(1-5 ; 6 ; 7 ; 8 ; 9)^{*}$ site $\left.\left(P^{*} H\right)\right]+$ year & 46 & 3935.9 & 4027.9 & 0.0 \\
\hline 19 & Age_7 & [age0; $(1-5 ; 6 ; 7 ; 8 ; 9)^{*}$ site $\left.\left(P^{*} H\right)\right]+$ year & 51 & 3928.5 & 4030.5 & 0.0 \\
\hline 20 & Lin_7 & [age0; $(1-5 ; 6 ; 7 ; 8 ; 9)^{*}$ site $\left.\left(P^{*} H\right)\right]+$ year & 46 & 3938.5 & 4030.5 & 0.0 \\
\hline 13 & Log_7 & [age $0 ;(1-5 ; 6 ; 7 ; 8 ; 9)^{*}$ site $\left.\left(P^{*} H\right)\right]+$ year & 46 & 3935.0 & 4027.0 & 0.0 \\
\hline 18 & Age_6 & [age $0 ;(1-5 ; 6 ; 7 ; 8 ; 9)^{*}$ site $\left.\left(P^{*} H\right)\right]+$ year & 50 & 3930.1 & 4030.1 & 0.0 \\
\hline 22 & Lin_6 & [age $0 ;(1-5 ; 6 ; 7 ; 8 ; 9)^{*}$ site $\left.\left(P^{*} H\right)\right]+$ year & 46 & 3939.1 & 4031.1 & 0.0 \\
\hline 10 & Log_6 & [age $0 ;(1-5 ; 6 ; 7 ; 8 ; 9)^{*}$ site $\left.\left(P^{*} H\right)\right]+$ year & 46 & 3934.7 & 4026.7 & 0.0 \\
\hline 17 & Age_5 & {$\left[\right.$ age $0 ;(1-5 ; 6 ; 7 ; 8 ; 9)^{*}$ site $\left.\left(P^{*} H\right)\right]+$ year } & 49 & 3930.3 & 4028.3 & 0.0 \\
\hline 12 & Lin_5 & [age0; $(1-5 ; 6 ; 7 ; 8 ; 9)^{*}$ site $\left.\left(P^{*} H\right)\right]+$ year & 46 & 3934.9 & 4026.9 & 0.0 \\
\hline 7 & Log_5 & [age $0 ;(1-5 ; 6 ; 7 ; 8 ; 9)^{*}$ site $\left.\left(P^{*} H\right)\right]+$ year & 46 & 3933.6 & 4025.6 & 0.0 \\
\hline 9 & Age_4 & [age $0 ;(1-5 ; 6 ; 7 ; 8 ; 9)^{*}$ site $\left.\left(P^{*} H\right)\right]+$ year & 48 & 3930.3 & 4026.3 & 0.0 \\
\hline 23 & Lin_4 & {$\left[\right.$ age $0 ;(1-5 ; 6 ; 7 ; 8 ; 9)^{*}$ site $\left.\left(P^{*} H\right)\right]+$ year } & 46 & 3939.5 & 4031.5 & 0.0 \\
\hline 8 & $\log _{-} 4$ & [age $0 ;(1-5 ; 6 ; 7 ; 8 ; 9)^{*}$ site $\left.\left(P^{*} H\right)\right]+$ year & 46 & 3934.2 & 4026.2 & 0.0 \\
\hline 14 & Age_3 & {$\left[\right.$ age $0 ;(1-5 ; 6 ; 7 ; 8 ; 9)^{*}$ site $\left.\left(P^{*} H\right)\right]+$ year } & 47 & 3933.2 & 4027.2 & 0.0 \\
\hline 32 & Lin_3 & [age $0 ;(1-5 ; 6 ; 7 ; 8 ; 9)^{*}$ site $\left.\left(P^{*} H\right)\right]+$ year & 46 & 3961.0 & 4053.0 & 0.0 \\
\hline 27 & Log_3 & [age $0 ;(1-5 ; 6 ; 7 ; 8 ; 9)^{*}$ site $\left.\left(P^{*} H\right)\right]+$ year & 46 & 3941.9 & 4033.9 & 0.0 \\
\hline 2 & Age_4* site & [age $0 ;(1-5 ; 6 ; 7 ; 8 ; 9)^{*}$ site $\left.\left(P^{*} H\right)\right]+$ year & 52 & 3912.9 & 4016.9 & 0.2 \\
\hline 5 & Log_5*site & [age $0 ;(1-5 ; 6 ; 7 ; 8 ; 9)^{*}$ site $\left.\left(P^{*} H\right)\right]+$ year & 47 & 3925.2 & 4019.2 & 0.1 \\
\hline 15 & Age_4* $4^{*}$ ex & [age $0 ;(1-5 ; 6 ; 7 ; 8 ; 9)^{*}$ site $\left.\left(P^{*} H\right)\right]+$ year & 52 & 3923.6 & 4027.6 & 0.0 \\
\hline 6 & Log_5*sex & [age $0 ;(1-5 ; 6 ; 7 ; 8 ; 9)^{*}$ site $\left.\left(P^{*} H\right)\right]+$ year & 47 & 3925.2 & 4022.9 & 0.0 \\
\hline 11 & Age_ $4^{*}$ sex ${ }^{*}$ site & [age $0 ;(1-5 ; 6 ; 7 ; 8 ; 9)^{*}$ site $\left.\left(P^{*} H\right)\right]+$ year & 60 & 3906.8 & 4026.8 & 0.0 \\
\hline 4 & Log_5*sex*site & {$\left[\right.$ age $0 ;(1-5 ; 6 ; 7 ; 8 ; 9)^{*}$ site $\left.\left(P^{*} H\right)\right]+$ year } & 49 & 3920.8 & 4018.8 & 0.1 \\
\hline 3 & Age_4*site $\left(P^{*}\right.$ sex $)$ & [age $0 ;(1-5 ; 6 ; 7 ; 8 ; 9)^{*}$ site $\left.\left(P^{*} H\right)\right]+$ year & 52 & 3914.0 & 4018.0 & 0.1 \\
\hline 1 & Log_5*site $\left(P^{*}\right.$ sex $)$ & [age $0 ;(1-5 ; 6 ; 7 ; 8 ; 9)^{*}$ site $\left.\left(P^{*} H\right)\right]+$ year & 47 & 3921.2 & 4015.2 & 0.5 \\
\hline
\end{tabular}

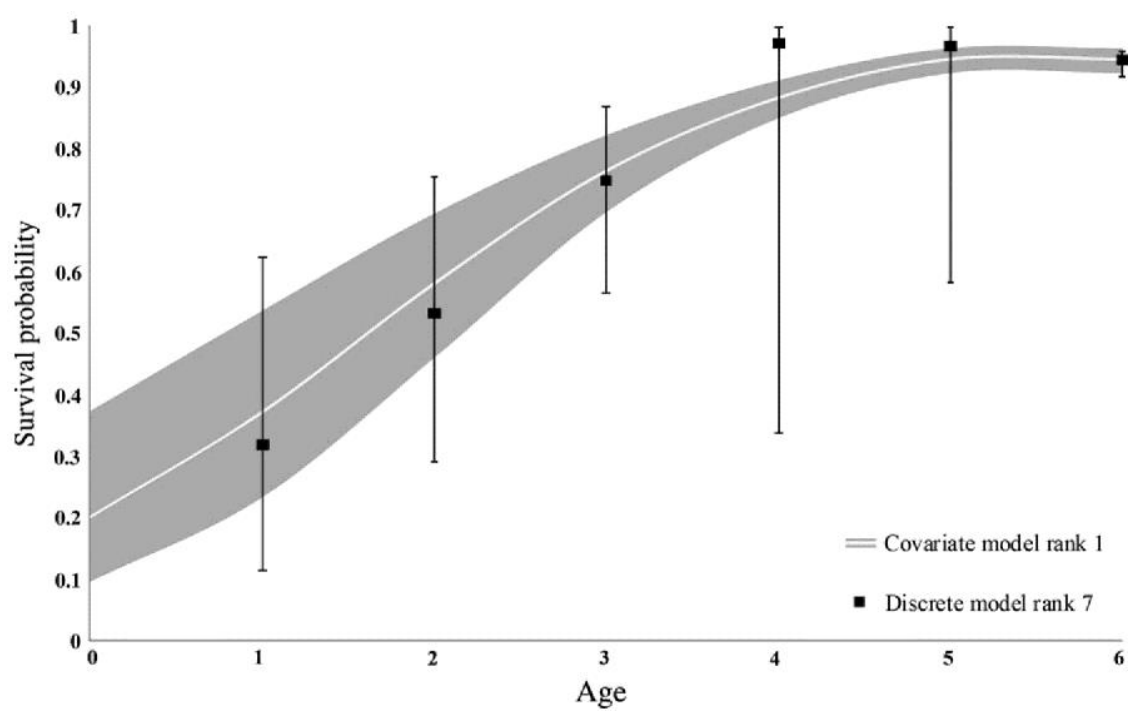

Figure 1 Estimated age-specific mean survival probabilities and 95\% CIs of young European pond turtles from Camargue, France obtained from a discrete model (Model rank 7, Table 5), superimposed against the estimates and $95 \%$ confidence spread of mean age-specific survival probabilities of the same population obtained from the best-fit covariate model (Model rank 1, Table 5). 


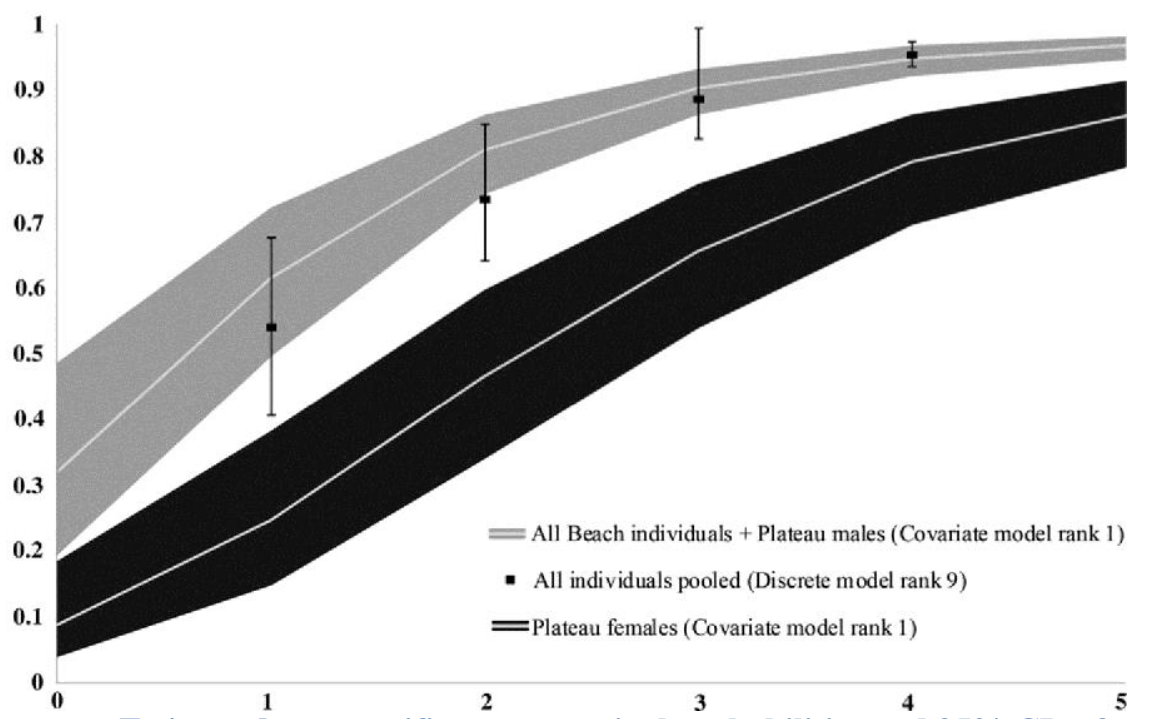

Figure 2 Estimated age-specific mean survival probabilities and 95\% CIs of young Hermann's tortoises from Golem Grad Island, Macedonia obtained from a discrete model (Model rank 9, Table 5), superimposed against the estimates and $95 \%$ confidence spread of mean age-specific survival probabilities of two separate cohorts (females from the Plateau, and both sexes from the Beach along with males from the Plateau) from the same population obtained from the best-fit covariate model (Model rank 1, Table 5).

pond turtle (Fig. 1). Most notably, the discrete models failed to estimate neonate survival due to the lack of marked neonates. The continuous models partially solve this issue by projecting the linear relationship of age and survival on neonate survival. Nevertheless, such predictions bear considerable uncertainty and should only be considered surrogates until the y can be supported by proper data collected on this age class. Attributed to the difficulty of capturing and recapturing elusive immature reptiles, researchers have often opted for indirect estimates of juvenile survival, such as catch-curve analyses (Table 1), or indirect mathematical approaches (Pike et al., 2008). The only robust study comes from a reintroduction program of western Hermann's tortoise adults allowing for all future recruits to be monitored - an exceptional case providing 19 years of data and precise discrete estimates (Fernàndez-Chacon et al., 2011); superimposing our eastern subspecies estimates reveals no differences between the taxa, and simply corroborates the idea that age-linear covariates helps streamline results without added bias. The following results thus seem encouraging; ultimately, substantial field effort followed by appropriate statistical approaches can pro-vide precise trait estimates, indispensable to matrix models underlying evolutionary ecology research, and efficient conservation policies.

Our results seemingly conform to existing literature (Table 1) - mean survival is lowest in neonates, yet never below $20 \%$, and it increases progressively with age (Fig. 1 and 2). Mitrus (2005) estimated surprisingly low survival values for neonate European pond turtles (0.11); whilst this might reflect the true situation, the author provides "minimal annual survivorships (numbers recaptured/number captured in previous year)", thus in practice actually describing a mix between survival and recapture rates. The age-survival relationships do not vary between our studied species despite their ecological differences and distant phylogenetic relatedness, possibly alluding a similar trend throughout this diverse clade (183 species in Crawford et al., 2014). Furthermore, several other long-lived vertebrates also exhibit a linear increase in age-specific survival (see for instance DeLong et al., 2017; Forslund \& P€art, 1995; Jorgenson et al., 1997; Schwarz \& Stobo, 2000; Shine \& Charnov, 1992). Different species reach their respective survival plateaus at different ages, often corresponding with maturity [2-3 years in bighorn sheep (Jorgenson et al., 1997 note that first reproduction usually occurs later), 4 years in grey seals (Schwarz \& Stobo, 2000), 7 years in Steller sea lions (Hastings et al., 2011), 5 years in California sea lions (DeLong et al., 2017)]. Both Hermann's tortoises and European pond turtles reach maturity between the ages of eight and 12 (six in Olivier, 2002; Rogner, 2009; Bertolero et al., 2011), yet arrive at a survival plateau earlier - at approximately four- to 5-yearsold (Fig. 1 and 2). This is likely a consequence of the final hardening of the carapace, which can bring obvious survival benefits prior maturity (Wilbur \& Morin, 1988). After maturity, European pond turtles from Camargue experience sex differences in survival (Olivier et al., 2010); nonetheless, our results overrule the possibility of it originating prior maturity. When immature neither species is obviously sexually dimorphic (Rogner, 2009; Bertolero et al., 2011), thus sex-specific habits or environmental pressures (e.g. sexual coercion in the Hermann's tortoise (Hailey \& Willemsen, 1999)] that could influence survival prior maturity are either elusive, or likely not present. In adult Hermann's tortoises sex-specific survival is not common apart from few populations with a bias in sex-ratio (Hailey \& Willemsen, 1999). Interestingly, the Golem Grad tortoise population has the highest reported bias in sex-ratio of this species, reaching its maximum on the Plateau where only 5\% of all adult individuals are female (Bonnet et al., 2016). Our best-fit model implies significantly lower survival of younger females from this locality from birth (Fig. 2). We hypothesize this result to be a consequence of male

coercion on the oldest females in our dataset, later projected onto the youngest age classes by the covariate. As such, this example is also a cautionary message on the use of covariates! Nevertheless, adult survival insight is needed, and more data for the discrete 
model to confirm these claims. Pin-pointing the age at which Plateau females are initially affected should be explored, but is beyond the scope of the current study.

The high variation in juvenile survival estimates that fail to give age- or at least stage-specific estimates in Table 1 is likely owed to the age-class that was predominant in the respective datasets. This mandates prudence when interpreting them in an evolutionary-ecological framework or applying them to conservation policies. Our results corroborate that immature survival increases with age in long-lived species, additionally suggesting it occurs rather steeply in chelonians. This is likely a consequence of ontogenic shifts in morphology, physiology and behaviour and therefore alterations in interactions with the environment (Golubovié, 2015). It would come as a surprise if shifts in age-specific survival do not translate to age-specific shifts in other aspect of a species' life history. Indeed, after the age of five Golem Grad tortoises experience ever-increasing agespecific body size variation (Fig. 1, Arsovski et al., 2018). Armed with a hard carapace they can reach a survival plateau and therefore enough confidence to start expressing individuality in their respective environments; interestingly, our models' preference to consider tortoise capture probabilities independently per age after the age of five corroborates this notion. Remarkably, behavioural shifts might follow this trend as well: Hermann's tortoises alter their antipredator behaviours after maturity (Golubovié, 2015). We suggest that future early life studies on species with a long immature phase should focus on age-specific considerations of other aspects of animals' life histories.

\section{Conclusion}

Reliable projection matrices based on accurate early life agespecific survival estimates are essential for fundamental and conservation purposes. We suggest a simple way of facilitating the process of obtaining these estimates even with sparse datasets. Systematic approaches to survey young animals should be unified, and the resulting (even sparse) age-specific sets of data can usefully be modelled using advanced statistical techniques. Continuous annual surveys of small and elusive animals might seem as an inefficient allocation of labour considering the output, but hopefully the advancements in direct methodological approaches will gradually change this notion.

\section{Acknowledgements}

We are thankful for field help from numerous collaborators, particularly Camille Buyck, Mitko Tasevski, Sébastien Ficheux, Sonja Nikolié, Marc Cheylan, Jean-Marie Ballouard, Elisabeth Rosecchi, Bogoljub Sterijovski and Rastko Ajtié, and for financial support from the Ministry of Education, Sciences and Technological Development (grant No. 173043) of Serbia, the Rufford Small Grants Foundation (grant no. 20915-1) and the

Tour du Valat Foundation. We thank the French Ministry of Environment and National Park 'Gali cica' (permit no. 03-246) for granting us work permits.

\section{References}

Arsovski, D., Tomovié, Lj, Golubovié, A., Nikolié, S.,

Sterijovski, B., Ajtié, R. \& Bonnet, X. (2018). When carapace governs size: variation among age classes and individuals in a

free ranging ectotherm with delayed maturity. Oecologia 186, 953.

Bertolero, A. (2002). Biologtia de la tortuga mediterrânea Testudo Hermanni aplicada a su conservacidn. PhD thesis,

Barcelona University.

Bertolero, A., Cheylan, M., Hailey, A., Livoreil, B. \& Willemsen, R.E. (2011). Testudo hermanni (Gmelin 1789) - Hermann's tortoise. Chelon. Res. Monogr. 5, 1.

Bertolero, A., Pretus, J.Lj \& Oro, D. (2018). The importance of including survival release costs when assessing viability in reptile translocations. Biol. Conserv. 217, 311.

Bjorndal, K.A., Bolten, A.B. \& Chaloupka, M.Y. (2003a). Survival probability estimates for immature green turtles Chelonia mydas in the Bahamas. Mar. Ecol. Prog. Ser. 252, 273.

Bjorndal, K.A., Bolten, A.B. \& Martins, H.R. (2003b). Estimates of survival probabilities for oceanic-stage loggerhead sea turtles (Caretta caretta) in the North Atlantic. Fish. Bull. 101, 732. Blamires, S.J., Spencer, R.J., King, P. \& Thompson, M.B. (2005).

Population parameters and life-table analysis of two coexisting freshwater turtles: are the Bellinger River turtle populations threatened. Wildl. Res. 32, 339.

Bonnet, X., Golubovié, A., Arsovski, D., Dordevié, S., Ballouard, J.M., Sterijovski, B., Ajtié, R. \& Tomovié, Lj (2016). A prison effect in a wild population: a scarcity of females induces homosexual behaviors in males. Behav. Ecol. 27, 1206.

Burnham, K.P. \& Anderson, D.R. (2002). Model selection and multimodel inference: a practical information-theoretic approach. New York, NY: Springer Verlag.

Cagle, F.R. (1939). A system for marking turtles for future identification. Copeia 1939, 170.

Canessa, S., Genta, P., Jesu, R., Lamagni, L., Oneto, F., Salvidio, S. \& Ottonello, D. (2016). Challenges of monitoring reintroduction outcomes: insights from the conservation breeding program of an endangered turtle in Italy. Biol. Conserv. 204, 128.

Castanet, J. (1988). Les méthodes d'estimation de l'âge chez les chéloniens. Mésogée 48, 21. 
Caswell, H. (2001). Matrix population models.

Construction, analysis, and interpretation. Sunderland, MA: Sinauer.

Chaloupka, M.Y. \& Limpus, C.J. (2002). Survival probability estimates for the endangered loggerhead sea turtle resident in southern Great Barrier Reef waters. Mar. Biol. 140, 267. Chaloupka, M. \& Limpus, C. (2005). Estimates of sex- and ageclassspecific survival probabilities for a southern Great Barrier Reef green sea turtle population. Mar. Biol. 146, 1251.

Charnov, E.L. (1990). On evolution of age at maturity and adult lifespan. J. Evol. Biol. 3, 139.

Choquet, R., Rouan, L. \& Pradel, R. (2009). Program E-SURGE: a software application for fitting multievent models. In Modeling demographic processes in marked populations: 845- 865. Thomson, D.L., Cooch, E.G. \& Conroy, M.J. (Eds). New York:

Springer Verlag.

Cites trade report on Testudo hermanni up to 2017. Available at: https://trade.cites.org

Congdon, J.D., Dunham, A.E. \& van Loben, Sels R.C. (1993). Delayed sexual maturity and demographics of Blanding's turtles

(Emydoidea blandingi): implications for conservation and management of long-lived organisms. Conserv. Biol. 7, 826.

Congdon, J.D., Dunham, A.E. \& van Loben Sels, R.C. (1994). Demographics of common snapping turtles (Chelydra serpentina) implications for conservation and management of long-lived organisms. Am. Zool. 34, 397.

Crawford, N. G., Parham, J. F., Sellas, A. B., Faircloth, B. C., Glenn, T. C., Papenfuss, T. J., Henderson, J. B., Hansen, M. H. and Simsion, W. B. 2014. A phylogenetic analysis of turtles. Mol. Phylogenet. Evol. 83: 250.

Cunnington, D.C. \& Brooks, R.J. (1996). Bet-hedging theory and eigenelasticity: a comparison of the life histories of loggerhead sea turtles (Caretta caretta) and snapping turtles (Chelydra serpentina). Can. J. Zool. 74, 291.

DeLong, I.R., Melin, S.R., Laake, J.L., Morris, P., Orr, A.J. \& Harris, J.D. (2017). Age- and sex-specific survival of California sea lions (Zalophus californianus) at San Miguel Island, California. Mar. Mammal. Sci. 33, 1097.

van Dijk, P.P., Iverson, J.B., Rhodin, A.G.J., Shaffer, H.B. \& Bour, R. (2014). Turtles of the world, 7th edition: annotated checklist of taxonomy, synonymy, distribution with maps, and conservation status. Chelon. Res. Monogr. 5, 329.

Dunham, A.E., Miles, D.B. \& Reznick, D.N. (1988). Lifehistory patterns in squamate reptiles. In Biology of the reptilie, Volume 16, ecology B: defense and life-history: 441-522. Gans, C. \& Huey, R.B. (Eds). New York: Alan R. Liss.

Enneson, J.J. \& Litzgus, J.D. (2008). Using long-term data and a stage-classified matrix to assess conservation strategies for an endangered turtle (Clemmys guttata). Biol. Conserv. 141, 1560.

Epperly, S. P., Snover, M. L., Braun-McNeill, J., Witzell, W. N., Brown, C. A., Csuzdi, L. A., Teas, W. G., Crowder, L. B. and Myers, R. A. (2001). Stock assessment of loggerhead sea turtles of the western North Atlantic. In NOAA Technical Memorandum NMFSSEFSC-455: 3-66. National Marine Fisheries Service Southeast Fisheries Science Center. Miami: US Department of Commerce.

Fernandez-Chac6n, A., Bertolero, A., Amengual, A., Tavecchia, G., Homar, V. \& Oro, D. (2011). Spatial heterogeneity in the effects of climate change on the population dynamics of a Mediterranean tortoise. Glob. Change Biol. 17, 3075.

Ficheux, S., Olivier, A., Fay, R., Crivelli, A., Besnard, A. \& Béchet, A. (2014). Rapid response of a long-lived species to improved water and grazing management: the case of

European pond turtle (Emys orbicularis) in the Camargue, France. J. Nat. Conserv. 22, 342.

Forslund, P. \& Pärt, T. (1995). Age and reproduction in birds- hypotheses and tests. Trends Ecol. Evol. 10, 374.

Frazer, N.B. (1987). Preliminary estimates of survivorship for wild juvenile loggerhead sea turtles (Caretta caretta). J. Herpetol. $21,232$.

Frazer, N.B., Gibbons, J.W. \& Greene, J.L. (1990). Life tables for a slider population. In Life-history and ecology of the slider turtle: 183-200. Gibbons, J.W. (Ed.). Washington DC: Smithsonian Institution Press.

Frazer, N.B., Gibbons, J.W. \& Greene, J.L. (1991). Life-history and demography of the common mud turtle Kinosternon subrubrum in South Carolina, USA. Ecology 72, 2218.

Fritz, U. \& Chiari, Y. (2013). Conservation actions for European pond turtles - a summary of current efforts in distinct European countries. Herpetol. Notes 6, 105.

Golubovié, A. (2015). Ontogenetic shift of antipredator behaviour in Hermann's tortoises. Behav. Ecol. Sociobiol. $69,1201$.

Hailey, A. \& Willemsen, R.E. (1999). Population density and adult sex ratio of the tortoise Testudo hermanni in Greece:

evidence for intrinsic population regulation. J. Zool. 251, 325.

Hastings, K.K., Jemison, L.A., Gelatt, T.S., Laake, J.L., Pendleton, G.W., King, J.C., Trites, A.W. \& Pitcher, K.W. (2011). Cohort effects and spatial variation in age-specific survival of Steller sea lions from southeastern Alaska. Ecosphere 2, 111.

Henry, P.Y., Nougarède, J.P., Pradel, R. \& Cheylan, M. (1999). Survival rates and demography of the Hermann's tortoise Testudo hermanni in Corsica, France. In Current studies in herpetology: 189-196. Miaud, C. \& Guyetant, G. (Eds). Paris: Société herpétologique de France.

Heppel, S.S. (1998). Application of life-history theory and population model analysis to turtle conservation. Copeia $1998,367$.

Heppel, S.S., Crowder, L.B. \& Crouse, D.T. (1996a). Models to evaluate headstarting as a management tool for long-lived turtles. Ecol. Appl. 6, 556.

Heppell, S.S., Limpus, C.J., Crouse, D.T., Frazer, N.B. \& Crowder, L.B. (1996b). Population model analysis for the loggerhead sea turtle, Caretta caretta, in Queensland. Wildl. Res. 23, 143.

IUCN (2017).IUCN Red list of threatened species. Version 2017-2. Available from: www.iucnredlist.org. Downloaded on 21 October 2017. 
Iverson, J.B. (1991). Patterns of survivorship in turtles (Testudines). Can. J. Zool. 69, 385.

Jorgenson, J.T., Festa-Bianchet, M., Gaillard, J.-M. \& Wishart, W.D. (1997). Effects of age, sex, disease, and density on survival of bighorn sheep. Ecology 78, 1019.

Keller, C., Dfaz-Paniagua, C. \& Andreu, A.C. (1998). Survival rates and causes of mortality of Testudo graeca hatchlings in southwestern Spain. J. Herpetol. 32, 238.

Klemens, M. W. (2000). Turtle conservation. Washington DC: Smithsonian Institution Press, 1-344.

Mitchell, J.C. (1988). Population ecology and life-history of the freshwater turtles Chrysemys picta and Sternotherus odoratus in an urban lake. Herpetol. Monogr. 2, 40.

Mitrus, S. (2002). Historia 'zyciowa 'zôł wia błotnego, Emys orbicularis — implikacje dla ochrony gatunkow długoz'yja cych. PhD Thesis, Jagiellonian University, Krak6w.

Mitrus, S. (2005). Headstarting in European pond turtles (Emys orbicularis): does it work? Amphibia-Reptilia 26, 333.

Mitrus, S. and Zemanek, M. (2004). Survivorship of the European pond turtle Emys orbicularis in central Poland. In

Proceedings of 3rd International Symposium on Emys orbicularis: 103-107. Kosice: Biol. Bratisl.

Olivier, A. (2002). Ecologie, traits d'histoire de vie et conservation d'une population de cistude d'Europe Emys orbicularis en Camargue. Master thesis, Montpellier II University.

Olivier, A., Barbraud, C., Rosecchi, E., Germain, C. \& Cheylan, M. (2010). Assessing spatial and temporal population dynamics of cryptic species: an example with the European pond turtle. Ecol. Appl. 20, 993.

Patricio, A.R., Velez-Zuazo, X., Diez, C.E., Van Dam, R. \& Sabat, A.M. (2011). Survival probability of immature green turtles in two foraging grounds at Culebra, Puerto Rico. Mar. Ecol. Prog. Ser. 440, 217.

Pfaller, J.B., Chaloupka, M., Bolten, A.B. \& Bjorndal, K.A. (2018). Phylogeny, biogeography and methodology: a metaanalytic perspective on heterogeneity in adult marine turtle survival rates. Sci. Rep. 8, 5852.

Pike, D.A., Pizzatto, L., Pike, B.A. \& Shine, R. (2008). Estimating survival rates of uncatchable animals: the myth of high juvenile mortality in reptiles. Ecology 89, 607.

Pledger, S., Pollock, K.H. \& Norris, J.L. (2003). Open capturerecapture models with heterogeneity: I. Cormack-Jolly-Seber Model. Biometrics 59, 786.

Pradel, R. (2005). Multievent: an extension of multistate capture-recapture models to uncertain states. Biometrics $61,442$.

Prévot-Julliard, A.-C., Lebreton, J.-D. \& Pradel, R. (1998). Reevaluation of adult survival of Black-headed Gull (Larus ridibundus) in presence of recapture heterogeneity. Auk 115, 85.

Rogner, M. (2009). European pond turtle Emys orbicularis. Frankfurt: Edition Chimaria.

Schwarz, C.J. \& Stobo, W.T. (2000). Estimation of juvenile survival, adult survival, and age-specific pupping probabilities for the female grey seal (Halichoerus gryprus) on Sable Island from capture-recapture data. Can. J. Fish Aquat. Sci. 57, 247.

Shine, R. \& Charnov, E.L. (1992). Patterns of survival, growth and maturation in snakes and lizzards. Am. Nat. 139, 1257.

Stubbs, D. (1995). Testudo hermanni in France. In Red data book on Mediterranean Chelonians: 94-102. Ballasina, D. (Ed.). Bologna: Edagricola.

Tuberville, T.D., Norton, T.M., Todd, B.D. \& Spratt, J.S. (2008). Long-term apparent survival of translocated gopher tortoises: a comparison of newly released and previously established animals. Biol. Conserv. 141, 2690.

Wilbur, H.M. (1975). The evolutionary and mathematical demography of the turtle Chrysemys picta. Ecology 56, 64.

Wilbur, H.M. \& Morin, P.J. (1988). Life-history evolution in turtles. In Biology of the reptilie, Volume 16, ecology B: defense and life-history: 387-440. Gans, C. \& Huey, R.B. (Eds). New York: Alan R. Liss.

Wilson, D.S. (1991). Estimates of survival for juvenile Gopher tortoises, Gopherus polyphemus. J. Herpetol. 25, 376. 